\section{Symptomatic portal biliopathy following cyanoacrylate injection for a bleeding duodenal varix}

We describe a patient with EHPVO who underwent cyanoacrylate (glue) injection for a bleeding duodenal varix and developed symptomatic portal biliopathy on immediate follow-up.

\section{Case report}

A 24-year-old man presented to our hospital in the year 2000 with haematemesis and melena.He underwent three sessions of endoscopic variceal band ligation for esophageal varices at 3-week intervals. Abdominal ultrasonography revealed a portal cavernoma, and normal size and echotexture of the liver. On regular endoscopic follow-up, there were eradicated oesophageal varices, a small gastric varix and a large duodenal varix in the duodenal bulb extending into its junction with the second part; there were no red colour signs (RCS). He was prescribed beta-blocker therapy.

In November 2008, he presented with a history of melena for 1 day. Upper endoscopy revealed eradicated oesophageal varices and the duodenal varix as before but with stigmata of recent bleed. He underwent endoscopic variceal band ligation of the duodenal varix. Follow-up endoscopy after 3 weeks revealed persistence of the duodenal varix. Two $\mathrm{mL}$ of N-butyl2-cyanoacrylate in $2 \mathrm{~mL}$ of lipiodol were injected into the duodenal varix. Endoscopy performed after 48 hours and 3 weeks showed obliterated duodenal varix.

Three months later, the patient presented with jaundice and fever with chills of 1-week duration. The total leukocyte count was $11,600 / \mathrm{cmm}, 80 \%$ being neutrophils. The total bilirubin was $8 \mathrm{mg} / \mathrm{dL}$; alanine and aspartate aminotransferases were $62 \mathrm{U} / \mathrm{L}$ and $60 \mathrm{U} / \mathrm{L}$, respectively; and alkaline phosphatase 599 (normal 40-140) U/L. Abdomen ultrasonography revealed bilobar intrahepatic biliary radical dilatation with multiple, round, shadowing, echogenic lesions measuring 8-10 mm across in the line of the common bile duct (CBD). The patient underwent endoscopic retrograde cholangiopancreatography (ERCP); fluoroscopy showed irregular linear radio-opaque shadows around the CBD and the liver hilum suggestive of lipiodol mixed cyanoacrylate presumably in the paracholedochal veins (Figure 1). Injection of the dye was withheld in view of suspected cholangitis. Air cholangiogram revealed two short strictures at the upper end of the CBD but no calculi. A diagnosis of portal biliopathy was considered; careful papillotomy was done; and a 10 Fr $15 \mathrm{~cm}$ straight biliary stent was placed (Figure 2). A cholangiogram after 3 months showed a short segment of eccenteric narrowing in the upstream bile duct (stricture) but disappearance of cyanoacrylate in the paracholedochal veins (Figure 3). Restenting was done with four 10 Fr $12 \mathrm{~cm}$ long biliary stents. Since then, he has undergone regular endoscopic stent exchange with four straight biliary stents being placed each time over the past 2 years. A stent-free trial was attempted, but he developed jaundice within 3 weeks, which necessitated repeat placement of biliary stents. Surgery is not being considered since there are no good shuntable veins.

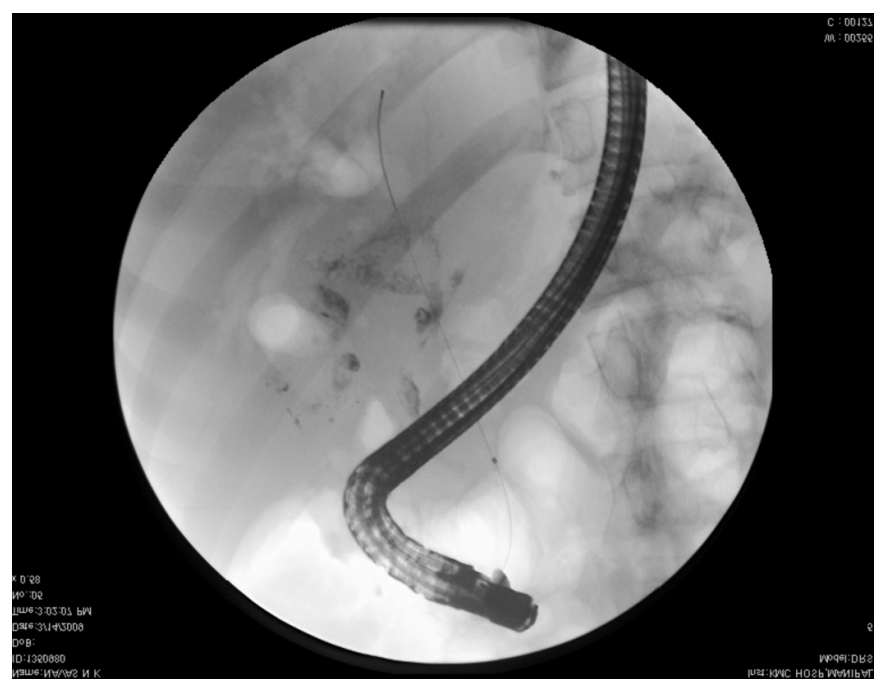

Figure 1: Paracholedochal cyanoacrylate with lipiodol cast seen at endoscopic retrograde cholangiopancreatography (ERCP). The air cholangiogram shows a dilated upstream biliary tree

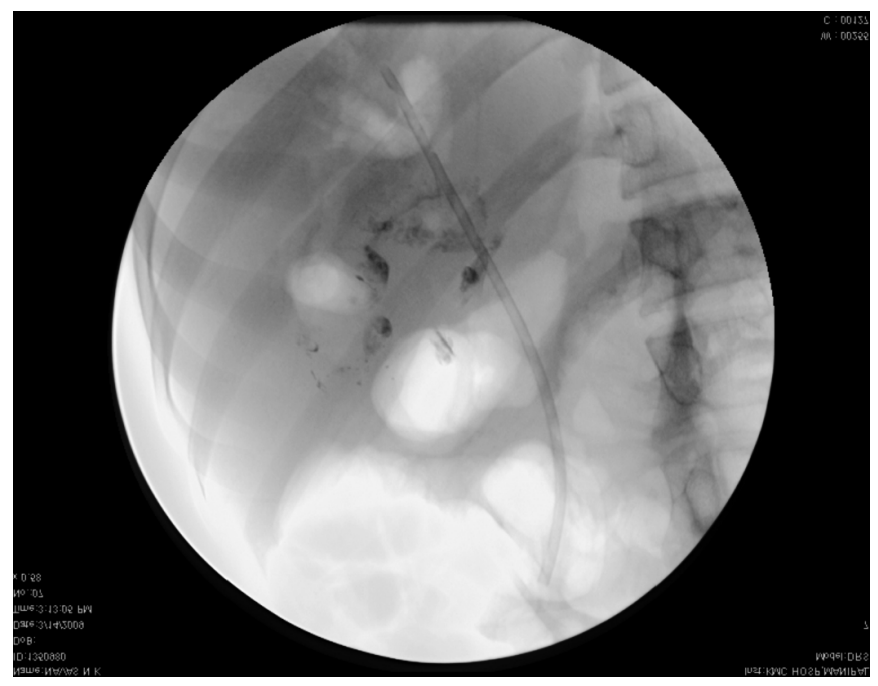

Figure 2: Bile duct stent in situ 


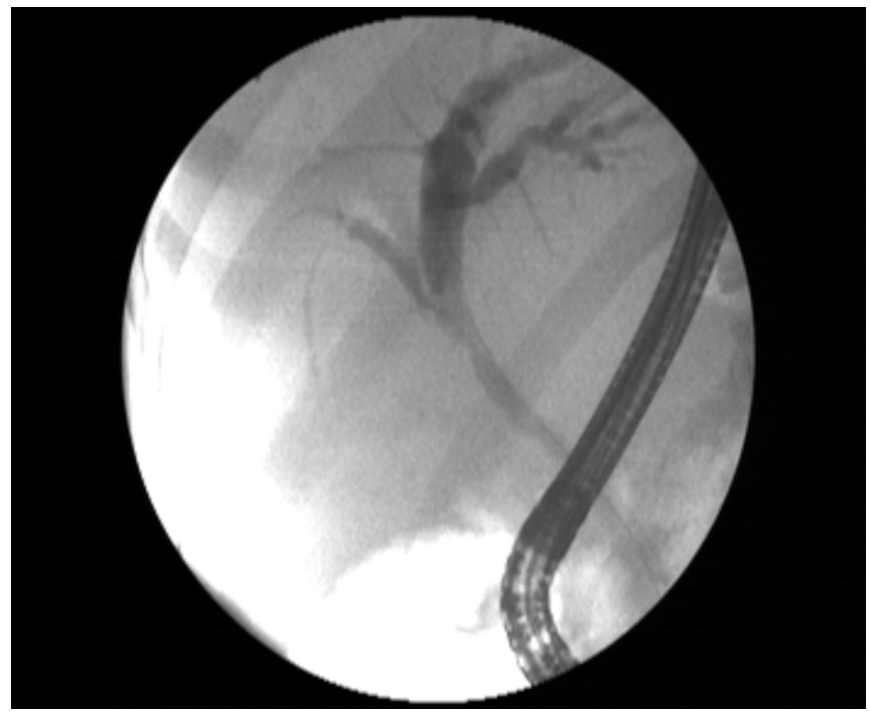

Figure 4: ERCP showing biliary stricture at the upper end with disproportionate left side biliary dilatation and disappearance of cyanoacrylate

\section{Discussion}

Duodenal varices result from retroperitoneal shunts caused by increased blood flow between the cystic branch of the superior mesenteric vein, superior and inferior pancreatic veins, and retroduodenal veins. Endoscopic cyanoacrylate injection has been shown to be a promising treatment option for bleeding

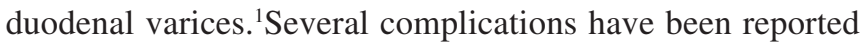
with the injection of cyanoacrylate into gastric varices, especially embolization of the glue into the portal, pulmonary, coronary and cerebral veins in up to $5 \%$ of cases. ${ }^{2,3}$ The risk of embolism appears to be related to the diameter of the vessel involved, pattern of blood flow, the volume injected and the rate of injection. ${ }^{2,3}$ Injecting cyanoacrylate without diluting it with lipiodol-thus using a lesser volume-might have decreased the spillage into paracholedochal varices.

The paracholedochal veins course parallel to the CBD and are connected to the gastric veins, the pancreaticoduodenal vein, the portal vein and to the liver. The postulated mechanisms of biliary stricture in portal biliopathy are mechanical compression of the biliary tree by the veins and ischaemic injury to the CBD. ${ }^{4,5}$

In our patient, biliopathy possibly resulted from ischaemic changes secondary to cyanoacrylate casts in the paracholedochal veins and collaterals. Another possibility is mechanical compression of the CBD by these casts. A third possibility is spillage of a small amount of cyanoacrylate directly into the CBD. The possibility of asymptomatic portal biliopathy predating the injection of cyanoacrylate cannot be ruled out, since a majority of patients with portal biliopathy reported in the literature remain asymptomatic. ${ }^{6}$ However, the absence of biochemical and sonographic evidence of the biliary obstruction before the injection therapy and sudden appearance of these after, along with the demonstration of radio-opaque casts, suggests a cause-effect relationship between the two. As biliary strictures persisted in our patient, even after $1 \frac{1}{2}$ years, by which time the casts had disappeared, ischaemia is the most likely mechanism involved. The possibility of spillage of cyanoacrylate into the CBD is less likely as the strictures are at the upper end of the CBD and they persist even after $1 \frac{1}{2}$ years of follow-up.

\section{GANESH BHAT DEEPAK SUVARNA \\ C. GANESH PAI}

Correspondence: Dr C. Ganesh Pai
Department of Gastroenterology \& Hepatology
Kasturba Medical College, Manipal University
Manipal 576104, Karnataka, India
Email: cgpai@yahoo.co.in

\section{References}

1. Liu Y, Yang J, Wang J, Chai G, Sun G, Wang Z, et al. Clinical characteristics and endoscopic treatment with cyanoacrylate injection in patients with duodenal varices. Scan J Gastroenterol. 2009;44:1012-16.

2. Seewald S, Sriram PV, Naga M, Fennerty MB, Boyer J, Oberti F, et al. Cyanoacrylate glue in gastric variceal bleeding. Endoscopy. 2002;34:926-32.

3. Suga T, Akamatsu T, Kawamura Y, Saegusa H, Kajiyama M, Nakamura N, et al. Actual behaviour of N-butyl-2-cyanoacrylate (histoacryl) in a blood vessel: a model of the varix. Endoscopy. 2002;34:73-7.

4. Chandra R, Kapoor D, Tharakan A, Chaudhary A, Sarin SK. Portal biliopathy. J Gastroenterol Hepatol. 2001;16:1086-92.

5. Dhiman RK, Behera A, Chawla YK, Dilawari JB, Suri S. Portal hypertensive biliopathy. Gut. 2007;56:1001-8.

6. Khuroo MS, Yatoo GN, Zargar SA, Javid G, Dar MY, Khan BA, et al. Biliary abnormalities associated with EHPVO. Hepatology. 1993;17:807-13.

\section{Neuromuscular and vascular hamartoma of small bowel with prominent inflammatory changes}

